\title{
LncRNA CDKN2B-AS1/miR-141/cyclin D network regulates tumor progression and metastasis of renal cell carcinoma
}

\author{
Pritha Dasgupta (1]', Priyanka Kulkarni ${ }^{1}$, Shahana Majid ${ }^{1}$, Yutaka Hashimoto ${ }^{1}$, Marisa Shiina ${ }^{1}$, Varahram Shahryari ${ }^{1}$, \\ Nadeem S. Bhat ${ }^{2}$, Laura Tabatabai ${ }^{1}$, Soichiro Yamamura', Sharanjot Saini ${ }^{3}$, Yuichiro Tanaka ${ }^{1}$ and Rajvir Dahiya ${ }^{1}$
}

\begin{abstract}
The molecular heterogeneity of renal cell carcinoma (RCC) complicates the therapeutic interventions for advanced metastatic disease and thus its management remains a significant challenge. This study investigates the role of the IncRNA CDKN2B-AS1 and miR-141-3p interactions in the progression and metastasis of kidney cancer. Human renal cancer cell lines (ACHN and Caki1), normal RPTEC cells, tissue cohorts, and a series of in vitro assays and in vivo mouse model were used for this study. An overexpression of CDKN2B-AS1 was observed in RCC compared to normal samples in TCGA and our in-house SFVAMC tissue cohorts. Reciprocally, we observed reduced expression of miR-141 in RCC compared to normal in the same cohorts. CDKN2B-AS1 shares regulatory miR-141 binding sites with CCND1 and CCND2 genes. Direct interactions of CDKN2B-AS1/miR-141/Cyclin D1-D2 were confirmed by RNA immunoprecipitation and luciferase reporter assays indicating that CDKN2B-AS1/miR-141/Cyclin D1-D2 acts as a ceRNA network in RCC. Functionally, attenuation of CDKN2B-AS1 and/or overexpression of miR-141 inhibited proliferation, clonogenicity, migration/invasion, induced apoptosis in vitro and suppressed tumor growth in xenograft mouse model. Further, overexpression of CDKN2B-AS1 is positively correlated with poor overall survival of RCC patients. Expression of miR-141 also robustly discriminated malignant from non-malignant tissues and its inhibition in normal RPTEC cells induced procancerous characteristics. CDKN2B-AS1 attenuation or miR-141 overexpression decreased CCND1/CCND2 expression, resulting in reduced RAC1/PPXN that are involved in migration, invasion and epithelial-mesenchymal transition. This study, for the first time, deciphered the role of CDKN2B-AS1/miR-141/Cyclin D axis in RCC and highlights this network as a promising therapeutic target for the regulation of EMT driven metastasis in RCC.
\end{abstract}

\section{Introduction}

Renal cell carcinoma (RCC) is one of the most common cancers in the USA accounting for nearly 14,830 deaths and 73,750 new cases in $2020^{1}$. Surgery is the first line of treatment resulting in successful resection and long-term disease-free status with an overall survival rate of more

\footnotetext{
Correspondence: Rajvir Dahiya (rdahiya@ucsf.edu)

${ }^{1}$ Department of Urology, Veterans Affairs Medical Center, San Francisco and University of California San Francisco, San Francisco, CA, USA

${ }^{2}$ Department of Surgery, University of Miami Miller School of Medicine, Miami, FL, USA

Full list of author information is available at the end of the article These authors contributed equally: Pritha Dasgupta, Priyanka Kulkarni Edited by E. Candi
}

than $60 \%$. However, in approximately $30 \%$ of localized RCC cases recurrence occurs with distant metastasis ${ }^{2}$. The obstinate nature of RCC to current treatment regimens is a primary cause of poor prognosis in patients with metastatic recurrence. Lack of sensitivity to both chemotherapy and immunotherapy makes therapeutic options difficult ${ }^{3-5}$. It is, therefore, of utmost importance to improve our understanding of RCC pathogenesis by identifying new biomarkers that lead to better prediction and therapeutic intervention of aggressive $\mathrm{RCC}^{6}$.

Emerging lines of evidence suggest that cancer aggressiveness is associated with epithelial-mesenchymal transition $(\mathrm{EMT})^{7}$. It is a well-orchestrated process involved in

\section{(c) The Author(s) 2020}

(c) Open Access This article is licensed under a Creative Commons Attribution 4.0 International License, which permits use, sharing, adaptation, distribution and reproduction cc) in any medium or format, as long as you give appropriate credit to the original author(s) and the source, provide a link to the Creative Commons license, and indicate if changes were made. The images or other third party material in this article are included in the article's Creative Commons license, unless indicated otherwise in a credit line to the material. If material is not included in the article's Creative Commons license and your intended use is not permitted by statutory regulation or exceeds the permitted use, you will need to obtain permission directly from the copyright holder. To view a copy of this license, visit http://creativecommons.org/licenses/by/4.0/. 
tumor invasion and metastasis comprising characteristic phenotypic changes through transition from polarized immotile epithelial cells to motile mesenchymal cells ${ }^{8}$. EMT changes in cellular morphology and migratory properties are governed by numerous factors ${ }^{9}$. Increase in mesenchymal properties accompanied by augmented expression of mesenchymal markers like N-cadherin, fibronectin, vimentin and matrix metalloproteinase (MMPs) and decreased expression of epithelial markers, like E-cadherin, $\alpha$-E-Catenin, claudin etc. ${ }^{10-13}$ are common EMT phenomena. Often the progression of cancer through EMT is significantly induced by the interaction of Cyclin-D with its binding partner, Cdk4 which act as transcriptional regulators controlling cell proliferation and migration ${ }^{14-16}$. It is well known that Cyclin-D regulates the rate-limiting step in cell cycle progression from G1 to $\mathrm{S}$ phase. Accumulating evidence also suggest that, abnormal Cyclin-D-Cdk4 over-expression promotes tumor growth and metastasis ${ }^{17}$, but how this correlates with tumor metastasis or controls cell adherence and invasion is poorly understood.

Reports show that non-coding RNAs are involved in the regulation of factors involved in $\mathrm{EMT}^{18}$. MicroRNAs (miRNAs), a naturally occurring class of small noncoding RNA molecules of 18-22 nucleotides long ${ }^{19}$, are known to regulate gene expression via both translational inhibition and mRNA degradation ${ }^{20}$ whereas, long noncoding RNAs (lncRNAs), with more than 200 nucleotides, can also act as regulators for tumor-suppressive miRNAs in different cancers $^{21-23}$. Recently, a class of IncRNAs have been categorized as competing endogenous RNA (ceRNA) which involves crosstalk among lncRNAs, mRNAs, and their shared miRNAs. Thus a novel regulatory mechanism is hypothesized suggesting that lncRNAs and mRNAs communicate with each other by competing for common miRNA response elements ${ }^{24-26}$.

In this context, we describe the novel role of IncRNA CDKN2B-AS1 and miR-141-3p (miR-141) in the regulation of Cyclin-D to govern the metastatic progression of RCC. To our knowledge, this is the first report to directly demonstrate that $C D K N 2 B-A S 1 / \mathrm{miR}-141$ interaction is a crucial component in RCC progression and metastasis through the Cyclin-D/Rac /paxillin pathway.

\section{Materials and methods}

\section{Cell lines and cell culture}

The normal RPTEC (ATCC number: CRL-4031) and renal cancer ACHN (ATCC number: CRL-1611) and Caki1 (ATCC number HTB-46) cell lines were purchased from the ATCC (Manassas, VA). These humanderived cell lines were authenticated by DNA shorttandem repeat analysis by ATCC. Cell line experiments were performed within 5-6 months of their procurement/ resuscitation. ACHN cells were cultured in MEM media,
Caki1 cells in and McCoy 5A medium, and RPTEC cells in DMEM:F12 Medium (ATCC ${ }^{\circledR} 30-2006^{\mathrm{TM}}$ ). All media were supplemented with $10 \%$ FBS and $1 \mathrm{X}$ antibiotics (penicillin and streptomycin). Cell lines were maintained at $37^{\circ} \mathrm{C}$ and humidified atmosphere of $5 \% \mathrm{CO}_{2}$.

\section{miRNA/siRNA transfections}

To induce overexpression or knockdown, cells were transiently transfected with either mirVana miRNA Mimic $(50 \mathrm{nmol} / \mathrm{L})$, or anti-miR miRNA inhibitor $(50 \mathrm{nmol} / \mathrm{L})$ (Thermo Fisher Scientific), and $30 \mathrm{nmol} / \mathrm{L}$ of siRNA (Sigma Aldrich) using Lipofectamine RNAi Max (Thermo Fisher Scientific) according to the manufacturers' protocol. To verify transfection efficiency, mirVana miRNA Mimic Negative Control \#1, miRNA inhibitor control and siRNA control were used respectively in each transfection experiment at the same concentration. All transfection experiments were carried out for $72 \mathrm{~h}$.

\section{Clinical specimens}

Formaldehyde-fixed-paraffin-embedded (FFPE) tissue specimens from patients undergoing radical nephrectomy were obtained from the San Francisco Veterans Affairs Medical Center (SFVAMC). Written informed consent was obtained from all patients and the study was in accordance with institutional guidelines (IRB approval no: 16-18555). All patient samples were pathologically confirmed for clear cell RCC (cc-RCC), and slides were reviewed by a board-certified pathologist for the identification of tumor foci and adjacent normal tissue. Apart from SFVAMC cohort, TCGA-KIRC, TCGA-KICH, TCGA-KIRP, ICGC, and GEO cohorts for RCC (from online databases) were also used to check the expression levels.

\section{RNA/miRNA extraction and quantitative real-time PCR (qRT-PCR)}

Total RNA was extracted from microdissected FFPE tissues and cell lines using miRNeasy FFPE and miRNeasy mini kits (Qiagen) respectively in accordance to manufacturer's instructions. Mature miRNA and mRNAs were assayed by qRT-PCR using QuantStudio 7 Flex-Real Time PCR System (Applied Biosystem) using Fast SYBR ${ }^{\circledR}$ Green Master Mix/TaqMan universal PCR master mix, probes and primers (Applied Biosystems Inc., Foster City, CA, USA) following manufacturer's protocol. Human GAPDH and RNU48 were used as endogenous controls, and relative expression of RNA/miRNA were calculated using comparative $\mathrm{Ct}$ (threshold cycle). Primer sequences are provided in Supplementary Table T1.

\section{DNA methylation analysis in-silico, in cell lines, and 5Aza- CdR treatment}

DNA hypermethylation of the miR-141 promoter in normal and RCC samples was first confirmed in the 
TCGA data-base using Wanderer software ${ }^{27}$. In order to confirm the methylation status of the miR-141 promoter in RCC cell lines, we extracted DNA from ACHN and Caki1 using DNeasy tissue kit (Qiagen). Sodium bisulphite modification was done using EZ DNA methylationGold kit (Zymo Research, Orange, CA, USA) following the manufacturer's protocol. Bisulfite-treated DNA was analyzed by methylation-specific quantitative polymerase chain reaction (MS-qPCR) with primer pairs specific for methylated and unmethylated regions of the miR141 promoter. MS-qPCR was performed as described ear$\operatorname{lier}^{28}$. For each sample, the percent of methylation was calculated by the difference of $\mathrm{Ct}$ in methylated sample $(\mathrm{Ct}-\mathrm{M})$ and $\mathrm{Ct}$ in unmethylated sample $(\mathrm{Ct}-\mathrm{U})$. The primers sequences are mentioned in Supplementary Table 1. ACHN and Cakil cells were treated daily with $20 \mu \mathrm{mol} / \mathrm{L}$ 5-AZA-deoxycytidine (5Aza-CdR) (Sigma-Aldrich) for $72 \mathrm{~h}^{29}$ and total RNA was isolated using a miRNeasy mini kit (Qiagen) to check miR-141 expression.

\section{Cell viability, clonability, migratory, invasion, and apoptosis assays}

Cell viability was measured at 24,48 , and $72 \mathrm{~h}$ using a CellTiter 96 Aqueous Solution Cell Proliferation Assay kit (Promega, Madison, WI) following the manufacturer's instructions. For colony formation assay, cells were seeded at a low density (1000 cells/plate) after $72 \mathrm{~h}$ of transfection and were allowed to grow until visible colonies were formed. Plates were then stained with giemsa followed by crystal violet and colonies were counted. Culture inserts of $8-\mu \mathrm{m}$ pore size (Transwell; Costar) were used for migration and invasion assay. Inserts were coated with Matrigel (BD Biosciences) $(100 \mu \mathrm{g} /$ well $)$ for invasion. Briefly, $72 \mathrm{~h}$ post-transfection, cells were counted and placed on inserts at $0.5 \times 10^{5}$ cells $/ \mathrm{ml}$ (for migration) and $1 \times 10^{5}$ (for invasion) in serum-free medium and were allowed to migrate/invade for $48-72 \mathrm{~h}$ at $37^{\circ} \mathrm{C}$. Cells migrated or invaded through the pores were fixed, stained with $0.05 \%$ crystal violet. Crystal violet was solubilized with methanol, and quantified at $540 \mathrm{~nm}$ by a kinetic microplate reader (Spectra MAX 190; Molecular Devices). FACS analysis for apoptosis was done $72 \mathrm{~h}$ post-transfection, using Annexin V-FITC and 7-AAD Kit (Beckman Coulter, Inc.) in accordance with the manufacturer's instructions. Cold PBS washed cells were resuspended in $1 \mathrm{X}$ binding buffer and stained with Annexin V-FITC/ 7AAD viability dye. After $30 \mathrm{~min}$ of incubation at room temperature in the dark, stained cells were analyzed using BD FACSVerse (BD Pharmingen).

\section{Dual-luciferase reporter assay}

The wild type (WT) and off-target (OT) luciferase reporter constructs were made by ligating annealed custom oligonucleotides containing putative target binding sites and corresponding nontarget /mutant sites into the pmiR-GLO reporter vector. Luciferase constructs $(0.1 \mu \mathrm{g})$ were cotransfected into ACHN and Caki1 cells along with $50 \mathrm{nmol} / \mathrm{L} \mathrm{miR-141} \mathrm{mimic} \mathrm{or} \mathrm{control-miR} \mathrm{using} \mathrm{trans-}$ fection reagent JetPrime (Polyplus-transfection, Illkirch, France). Luciferase activities were measured using the Dual-luciferase assay (Promega, Madison, WI) $48 \mathrm{~h}$ post transfection. Relative luciferase activity was calculated by normalizing Firefly luciferase to Renilla luminescence.

\section{RNA immunoprecipitation (RIP) assay}

Simultaneous binding of miR-141 to lncRNA and mRNA was confirmed by RIP assay. An imprint RIP kit was used following the manufacturer's protocol (SigmaAldrich, St. Louis, MO, USA). IgG (control) and Ago2 antibodies were used for immunoprecipitation. The immunoprecipitated RNA fraction was reverse transcribed to cDNA using High capacity cDNA reverse transcription kit (Thermo Fisher). Fold enrichment of lncRNA and mRNA to Ago2 with respect to IgG was calculated using quantitative RT-PCR.

\section{Western blot and immunofluorescence analysis}

Total protein extraction was performed as described previously ${ }^{18}$. Proteins were then separated by NuPAGE 4-12\% Bis-Tris Protein Gels (Invitrogen) and subsequently transferred onto nitrocellulose membrane. Resulting blots were blocked using Odyssey blocking buffer (LI-COR) and subsequently probed with primary and secondary antibodies. Blots were scanned using an Odyssey Infrared Imaging System Scan and quantification was carried out with the LI-COR Odyssey® scanner and software (LI-COR Biosciences). The primary antibodies used are listed in Supplementary Table 2. For immunofluorescence, transiently transfected ACHN and Caki1 cells were fixed in $4 \%$ paraformaldehyde for $15 \mathrm{~min}$ followed by blocking (1X PBS/5\% normal goat serum/ $0.3 \%$ Triton $\mathrm{X}-100)$ for $1 \mathrm{~h}$ at room temperature. Cells were then incubated overnight in 1:100 fold diluted primary antibody at $4{ }^{\circ} \mathrm{C}$. Cells were then reprobed with 1:300 fold diluted secondary antibody for $2 \mathrm{~h}$ and counter stained with $0.5 \mu \mathrm{g} / \mathrm{ml}$ of $4^{\prime}$,6-diamidino-2-phenylindole (DAPI) for $5 \mathrm{~min}$. Cells were then mounted on a slide using prolong gold anti-fade reagent. Images were captured using Zeiss microscope (model: Axio Imager.D2).

\section{Renal cancer xenografts}

We studied the anti-tumorigenic effects of miR-141 in established tumors using a renal cancer xenograft nude mouse model as previously described ${ }^{6,30}$. Male nude mice (4-5 week-old, $n=10$; Charles River Lab) were subcutaneously injected with $1 \times 10^{7}$ Caki1 cells. Once palpable tumors were formed, mice were randomized in two groups for the treatment and control groups (five in 
each). Synthetic miRNA (miR-141 mimic/miR-CON) of $6.25 \mu \mathrm{g}$ was complexed with $1.6 \mu \mathrm{L}$ siPORTamine transfection reagent (Ambion) in $50 \mu \mathrm{L}$ PBS, and delivered intratumorally in 3-day intervals. Tumor volume was calculated according to the formula $\left\{(x)^{2} * y\right\} / 2$ where $x<y$ ( $x=$ width; $y=$ length). Experiments were terminated 3 days after the last treatment (day 38). Tumor measurements and statistical analysis were performed by researchers who were blinded for the control and treatment groups. All animal care was in accordance with institutional guidelines (IACUC approval no: 16-004).

\section{Statistical analysis}

All quantified data represents an average of at least three independent experiments or as indicated. Statistical analyses were performed using GraphPad Prism 7 and MedCalc. Error bars represent \pm standard error mean (S.E.M). The Mann-Whitney $U$ test was used to assess the difference between miRNA expressions in tumor/ normal adjacent tissue. Significant differences between the groups were determined using the Student's $t$-test. All tests were performed either one tailed or two tailed and results were considered statistically significant at $P \leq 0.05$. Receiver operating curves (ROC) were performed to evaluate the potential of miR-141 to differentiate between malignant and non-malignant samples using MedCalc software showing area under curve (AUC) and 95\% confidence interval. Kaplan-Meier analyses for overall survival with respect to miR-141 methylation levels in TCGA-KIRC cohort were generated using software "EZR" (https://doi.org/10.1038/bmt.2012.244). Tumor measurements and statistical analyses for all experiments were performed blindly for the control and treatment groups.

\section{Results}

IncRNA CDKN2B-AS1 is oncogenic and is a direct target of miR-141

Initially, we found $C D K N 2 B-A S 1$ is an oncogenic lncRNA in RCC based on TCGA (Fig. 1a), ICGC and GEO databases (Fig. S1). TCGA cohort also revealed that high $C D K N 2 B-A S 1$ expression increases from lower grade and stage to higher grade and stage (Fig. 1b). Moreover, higher expression is significantly $(p<0.0001)$ correlated to overall survival (Fig. 1c). In agreement with these data cohorts, significantly higher $C D K N 2 B-A S 1$ expression was also seen in RCC cell lines ACHN, Caki-1 as compared to normal RPTEC (Fig. 1d) and SFVAMC cohort (Fig. 1e). Patient and tumor characteristics are summarized in Supplementary Table T3. ROC analysis shows an AUC of $0.765(P<0.001 ; 95 \% \mathrm{CI}=0.684-0.833)$ (Fig. 1f) suggesting the diagnostic potential of CDKN2B-AS1 to discriminate between normal and tumor tissues. We used computational algorithms and identified putative miR-141 binding sites in the CDKN2B-AS1 sequence (Fig. 1g). To examine potential miR-141/CDKN2B-AS1 interaction experimentally, we performed luciferase reporter assay. Both ACHN and Cakil cells co-transfected with miR-141 and $C D K N 2 B-A S 1$ wild type binding site revealed a consistent reduction of luciferase activity suggesting that miR-141 directly interacts and regulates CDKN2B-AS1 (Fig. 1h). Thus, all these data suggest that clinically important $C D K N 2 B-A S 1$ is an oncogenic lncRNA in RCC and is a novel target of miR-141.

\section{CDKN2B-AS1 inhibition by siRNA suppresses tumorigenicity in RCC}

Transient transfection of ACHN and Cakil cells with $C D K N 2 B-A S 1$ siRNAs for $72 \mathrm{~h}$ showed significant reduction in CDKN2B-AS1 expression (Fig. 2a). CDKN2B-AS1 knockdown in both cell lines significantly inhibited cell proliferation (Figs. 2b, S2A), and clonogenic survival (Figs. 2c, S2B) with a significant increase in apoptosis (Fig. 2d, e). Decreased cell migration/invasion (Figs. 2f, S2C, D) with simultaneous changes in EMT markers such as an increase in epithelial markers ( $\alpha$-E-Catenin and claudin) and decrease in mesenchymal markers (vimentin and fibronectin) were also observed (Figs. 2g, S3).

\section{Expression of miR-141 and its clinical importance in renal carcinoma}

Since our results confirmed $C D K N 2 B-A S 1$ as a direct target of miR-141, we examined miR-141 status and clinical importance in RCC. Expression of miR-141 was significantly down-regulated in RCC cell lines (Fig. 3a) and in tumor samples (Fig. 3b-e) compared to normal cell line and samples. Lower expressions significantly decreased with increasing grade, stages and in metastatic compared to non-metastatic tumors (Fig. 3c-e). Patient and tumor characteristics are summarized in Supplementary Table T3. ROC analysis showed an AUC of 0.897 $(P<0.001 ; 95 \% \mathrm{CI}=0.833-0.943)$ (Fig. 3f) suggesting that miR-141 can be used as a potential diagnostic parameter to discriminate between normal and tumor tissues.

\section{Epigenetic regulation of the miR-141 locus}

We identified a genomic site rich in CpG island located upstream of the miR-141 in chromosome 12p13. In the TCGA cohort, we observed hypermethylation of miR-141 promoter in tumor tissues as compared to normal (Figs. 3g, S4A) which is significantly associated with poor patient survival (Fig. 3h). Similarly, RCC cell lines ACHN and Cakil also showed hypermethylation compared to normal RPTEC cells (Fig. 3i). Further, we treated ACHN and Caki1 cell lines with demethylating agent 5-Aza-CdR and observed decrease in methylation (Fig. S4B) with concomitant increase in miR-141 expression (Fig. 3j) indicating possible epigenetic regulation. A significant decrease in the expression of methylation regulatory 


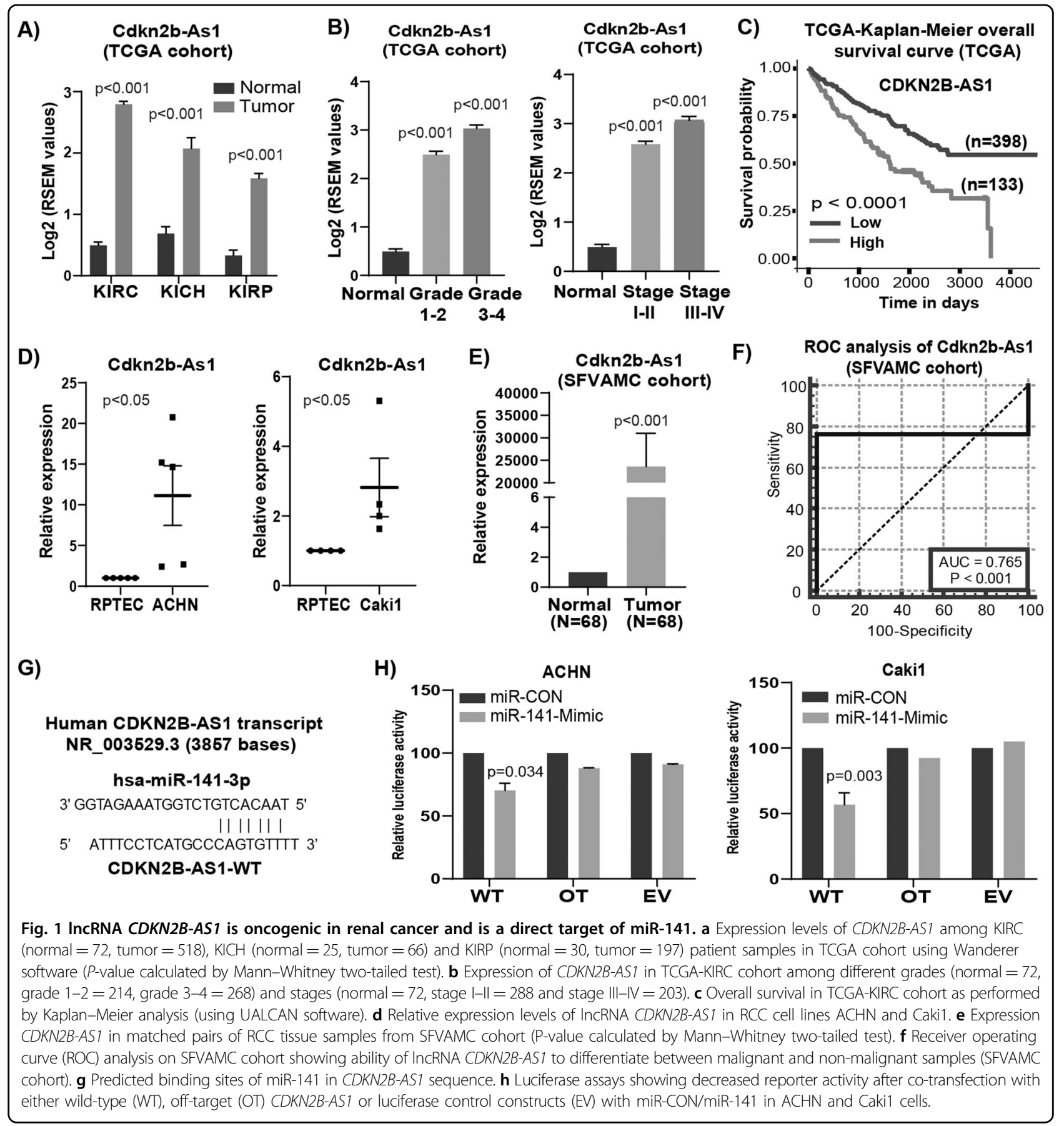

genes such as DNMTl, DNMT3a, and DNMT3b were also noted after 5-Aza-CdR treatment compared to control (DMSO) in both ACHN and Cakil cell lines ${ }^{31}$.

miR-141 over-expression phenocopies functional effects obtained with CDKN2B-AS1 inhibition in vitro and suppresses tumorigenicity and in vivo

We sought to determine if $C D K N 2 B-A S 1$ causes its anti-tumorigenic effects through miR-141. We checked the effect of miR-141 overexpression in RCC cells. Transient transfection of miR-141 mimic in ACHN and Caki1 cells for $72 \mathrm{~h}$ led to over expression of miR-141 compared to control (miR-CON) (Fig. 4a). Also, overexpression of miR-141 significantly reduced CDKN2B$A S 1$ expression (Fig. 4b) indicating a reciprocal correlation between miR-141 and CDKN2B-AS1. A significant decrease in cell proliferation over time (Fig. 4c) and marked decrease in clonogenicity (Fig. 4d) 


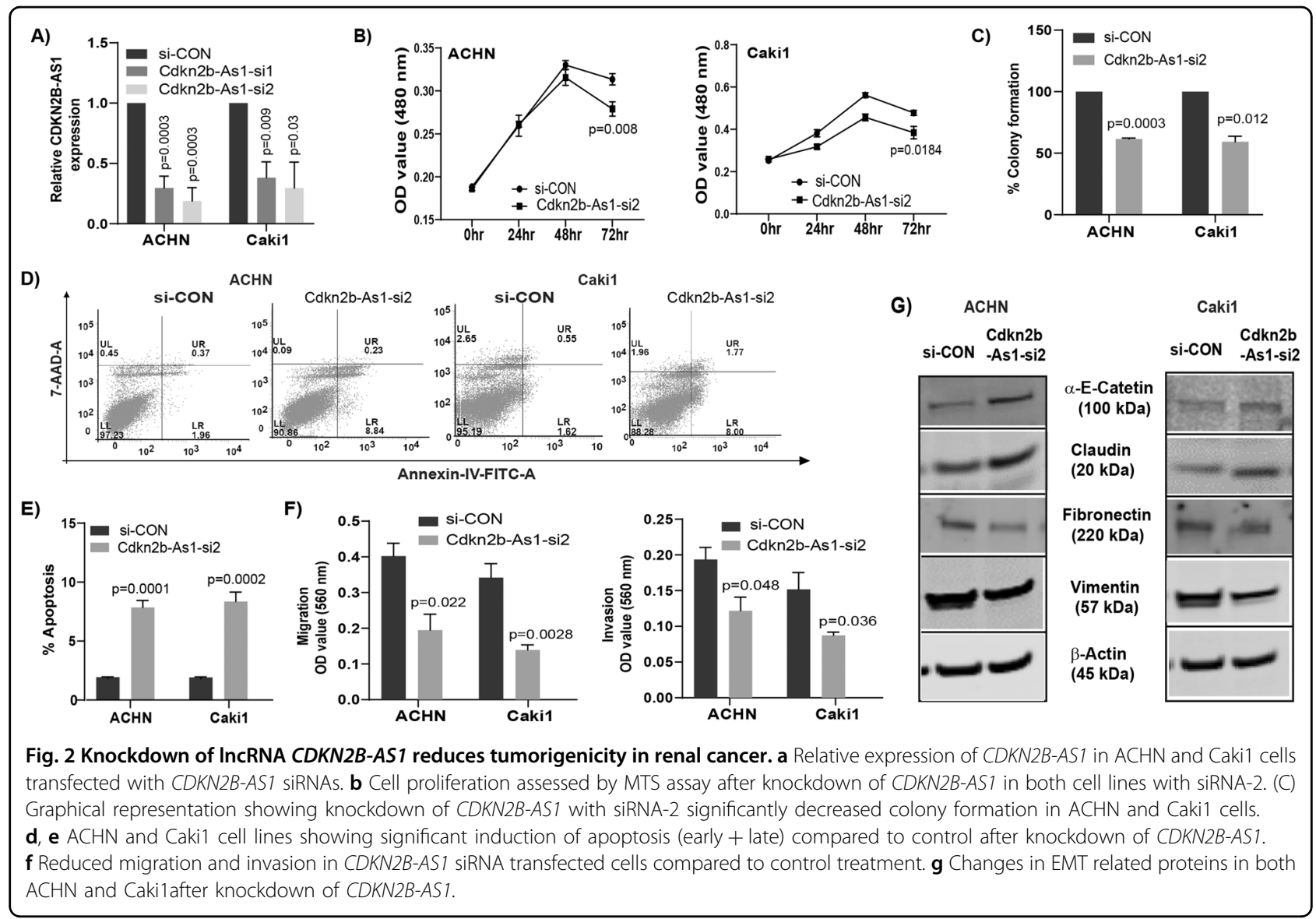

compared to controls were also observed. Further, we studied the therapeutic potential of miR-141 in a mouse xenograft model. A significant decrease in tumor growth was observed by intratumoral delivery of miR-141 mimic compared to control over the course of experiment. Average tumor volume in the control group was $1548.4 \mathrm{~mm}^{3}$ compared to $504.7 \mathrm{~mm}^{3}$ in mice that received miR-141 mimic (Fig. 4e). In addition, miR-141 over-expression significantly induced apoptosis with a concomitant decrease in the viable population in both RCC cell lines compared to control (Fig. 5a). This pro-apoptotic role was supported by the induction of cleaved caspase-3, cleaved poly-ADPribose polymerase (PARP), an increase in BAX, and a decrease in $\mathrm{BCl}_{2}$ at protein levels (Fig. 5b). A significant decrease in migration (Fig. 5c) and invasion (Fig. 5d) was also observed in both RCC cell lines with miR-141 overexpression. We also examined EMT markers as change in migration and invasion are directly associated with EMT. Our results showed an increase in epithelial markers $\alpha$-E-catenin and claudin with concomitant decrease in mesenchymal markers fibronectin and vimentin at both protein (Fig. 5e) and mRNA
(Fig. S5) levels. Taken together, overexpression of miR141 phenocopies the functional effects of $C D K N 2 B$ $A S 1$ inhibition in vitro and tumor growth suppression effects in vivo.

\section{miR-141/CDKN2B-AS1 interaction negatively regulates Cyclin-D and its downstream effectors in RCC}

Like CDKN2B-AS1, cyclin-D1/cyclin-D2 are also oncogenic in RCC (Fig. S6); and are direct targets of miR141. As discussed earlier, lncRNAs can act as ceRNAs to carry out their regulatory functions ${ }^{32-35}$. We observed that CDKN2B-AS1 shared regulatory miR-141 binding sites with cyclin-D1/cyclin-D2 (Fig. 6a), and thereby sponges miR-141 allowing cyclin-D1/D2 to be expressed in tumors. To determine potential miR-141/CDKN2B$A S 1 /$ Cyclin-D interaction experimentally, we performed RIP assay. Both ACHN and Cakil cells over expressing miR-141 revealed significant enrichment of cyclin-D1, cyclin-D2, and CDKN2B-AS1 with Ago2 as compared to IgG control (Fig. 6b). Moreover, decreased luciferase activity also confirmed direct binding of miR-141 to cyclin-D in miR-141 overexpressing ACHN and Caki1 cells compared to controls (Fig. 6c). We also found 


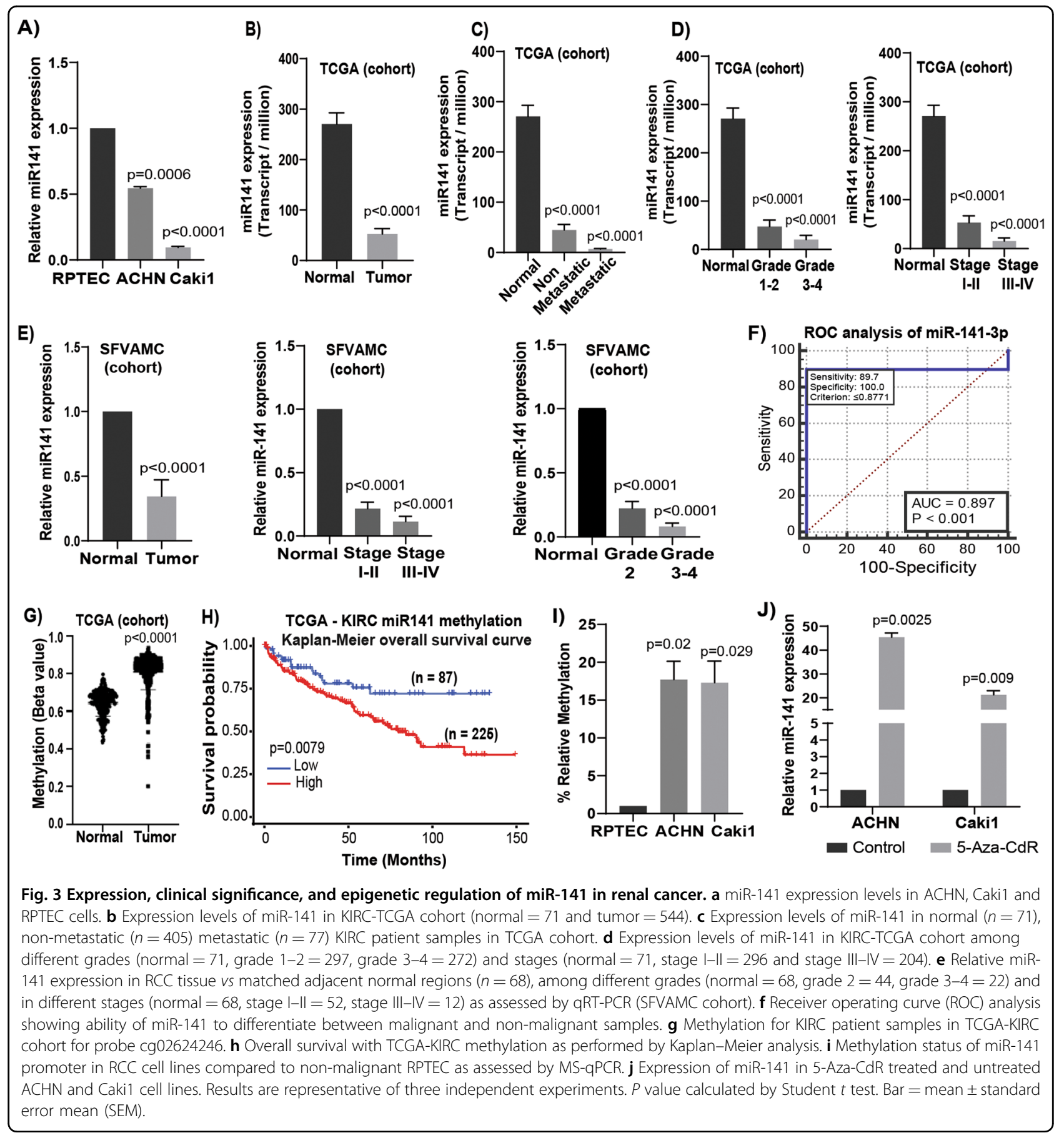

that overexpression of miR-141 or inhibition of $C D K N 2 B-$ AS1 significantly decreased cyclin-D1/D2 expression at both the mRNA (Fig. 6d, e) and protein levels (Figs. 6f-h, S8A, B). This effect was significantly attenuated by miR141 inhibitor (Fig. S7), indicating that cyclin-D expression is dependent on the interaction between miR-141 and $C D K N 2 B-A S 1$. We further observed a decrease in Rac1, a small GTPase, and a reduction in the phosphorylation of paxillin, a focal adhesion protein, at mRNA levels (Figs. S3, S5) and protein levels (Figs. 6g, I-j, S8C-F) in both miR-141 overexpressed and CDKN2B-AS1 inhibited RCC cell lines which in turn are involved in regulating cellular migration/invasion. Cumulatively, these results indicate that suppression of CDKN2B-AS1 by miR-141 inhibits renal cell proliferation, invasion and migration by inhibiting cyclin-D, Rac1, and phosphorylation of paxillin. 


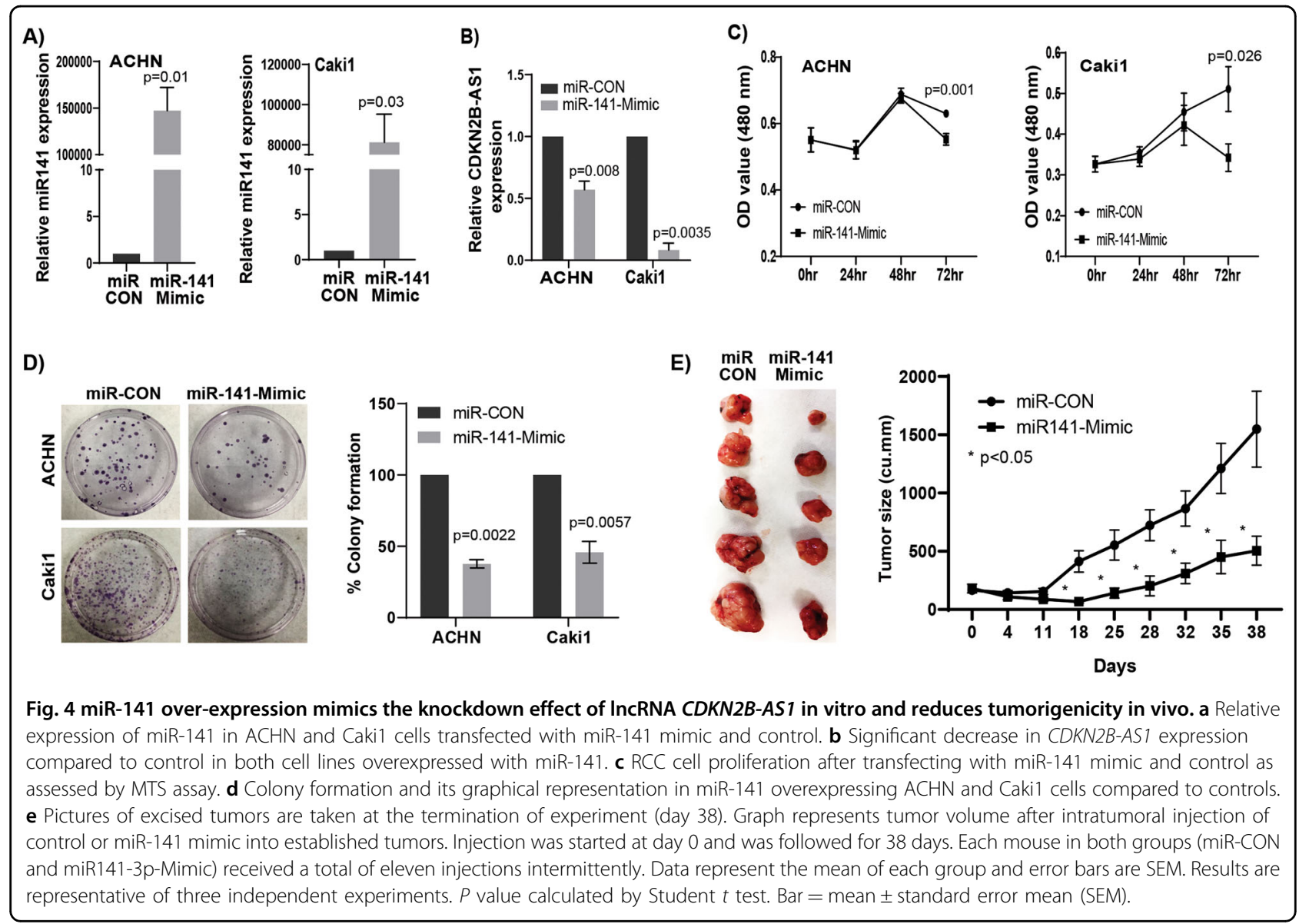

\section{Attenuation of miR-141 exerts tumorigenic attributes in normal RPTEC cells}

We next determined whether attenuation of miR-141 induces tumorigenic characteristics in normal RPTEC cells by targeting $C D K N 2 B-A S 1$ and cyclin-D. Transient transfection of miR-141 inhibitor indeed showed a significant decrease in miR-141 expression (Fig. 7a) and an increase in $C D K N 2 B-A S 1$ expression (Fig. 7b) along with other pro-cancerous phenotypes such as increased cell proliferation (Fig. 7c), colony formation (Fig. 7d), migration and invasion (Fig. 7e) as compared to controls. Additionally, a significant increase in cyclin-D1, cyclinD2, rac1, and paxillin (Pxn) expressions were observed in miR-141 inhibited RPTEC cells (Fig. 7f). A noticeable increase in pro-metastatic fibronectin and vimentin with a concomitant decrease in anti-metastatic claudin and $\alpha$-E-catenin genes were also observed in miR-141 inhibited RPTEC cells compared to controls (Fig. 7g).

\section{Discussion}

Prior studies have shown the regulatory role of noncoding RNAs in tumorigenesis especially in the EMT pathway leading to cancer aggressiveness. $C D K N 2 B-A S 1$, also known as $A N R I L$, is located at chromosome $9 \mathrm{p} 21$. $C D K N 2 B-A S 1$ is reported to be upregulated in tumor tissues and function as an oncogenic lncRNA in pancreatic, ovarian and laryngeal squamous cell carcinoma ${ }^{36-38}$. Human miR-141 is located at chromosome $12 \mathrm{p} 13.31$ and is transcribed from a miR-200 family cluster. Interestingly, expression of miR-141 is controversial since it exhibits either oncogenic ${ }^{39-41}$ or tumor suppressive roles $^{42-44}$ in specific types of cancer. The prime goal of the present study was to understand the role of $C D K N 2 B-A S 1 / \mathrm{miR}-141$ interactions in regulating $\mathrm{RCC}$ progression and metastasis.

In this study, we identify $C D K N 2 B-A S 1$ to be a crucial oncogenic lncRNA that plays an important role in renal carcinogenesis. $C D K N 2 B-A S 1$ is significantly overexpressed in RCC and the expression increases from lower to higher grades and stages. LncRNA CDKN2B-AS1 directly interacted with miR-141 as it was found to be a novel target of miR-141. In contrast to CDKN2B-AS1, we observed significant attenuation of miR-141 expression in RCC cell lines and tumor samples compared to normal 


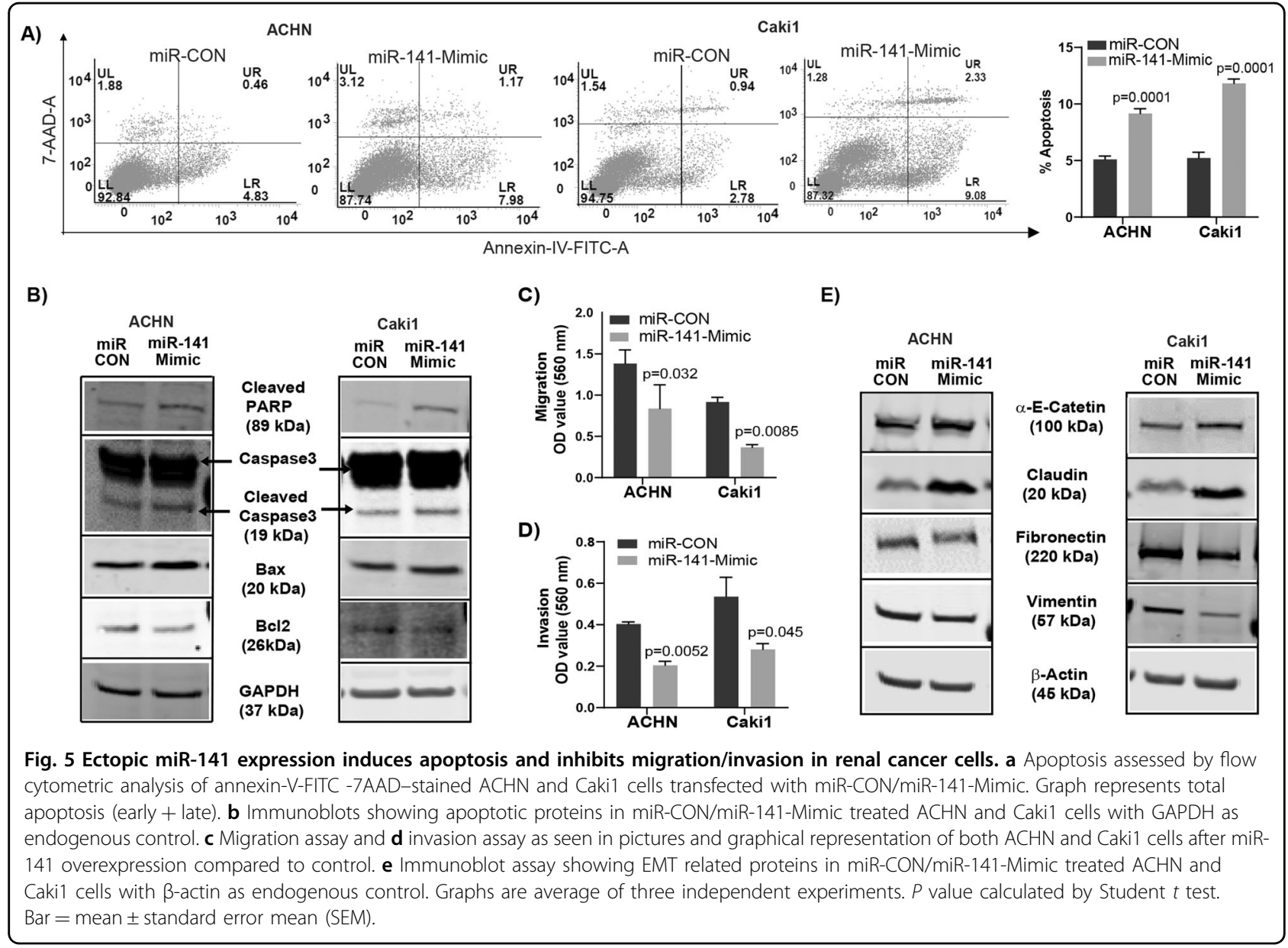

cell line or matched normal samples. As it is known that extensive DNA hypermethylation of CpG islands is highly correlated to activation of cancer-specific genes ${ }^{45}$, we checked the methylation status of miR-141 in normal and RCC tissues. Interestingly, in-silico analysis showed the presence of $\mathrm{CpG}$ island in the promoter region of miR141 and we also found hypermethylation of miR-141 in TCGA samples as compared to normal. This hypermethylation is also found to be significantly associated with poor survival of patients. Similar results were also observed in RCC cell lines compared to a normal RPTEC cell line. Functionally, inhibition of CDKN2B-AS1 and/or over-expression of miR-141 significantly inhibits the tumorigenic characteristics such as cell proliferation, clonogenicity, migration and invasion whereas induces anticancer apoptotic phenotype in RCC in vitro. In vivo data show suppression of tumor growth by miR-141 overexpression. Conversely, attenuation of miR-141 in normal RPTEC cells induced precancerous characteristics indicated by increased proliferation, migration and invasion.

From a clinical point of view, non-coding RNAs signatures are powerful tools for early cancer diagnosis making them attractive candidates as diagnostic and prognostic biomarkers ${ }^{18,46,47}$. Our results revealed that higher expression of $C D K N 2 B-A S 1$ is positively correlated with poor overall survival probability of RCC patients indicating its prognostic capability. CDKN2B-AS1 can also discriminate normal from tumor samples showing its diagnostic potential. Similarly, miR-141 expression can also robustly distinguish between cancerous from noncancerous samples and hence has potential to be a diagnostic biomarker for RCC. Collectively, these results highlight the biomarker potential of CDKN2B-AS1/miR141 expression in RCC, although it needs to be confirmed in a larger independent sample cohort.

Interestingly, we found that like lncRNA CDKN2B-AS1, cyclin-D1/D2 are also direct targets of miR-141. Accruing data proposes a new role for lncRNAs by pairing and sequestering specific miRNAs to protect repression of target mRNAs $^{32-35}$. Our in-silico analysis showing CDKN2B-AS1 shares regulatory miR-141 binding sites with cyclin-D1-D2 which was confirmed by luciferase and RIP assay. Cyclin-D1/D2 are also oncogenic in most cancers including RCC and play role in progression and metastasis ${ }^{15,48,49}$. Apart from the cell cycle regulation role 


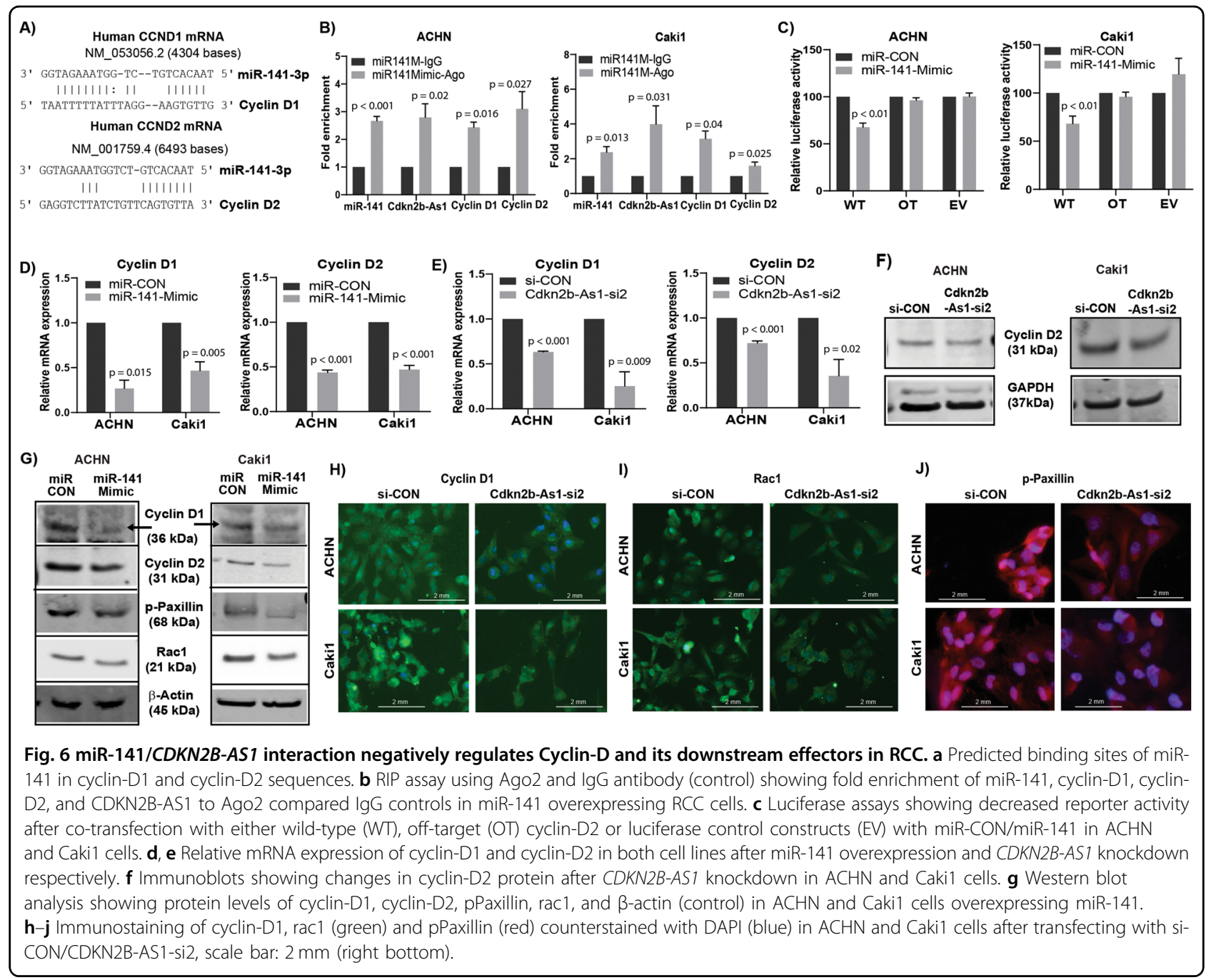

of cyclin-D, it is also important in maintaining cellular adhesion, migration and metastasis through phosphorylation of paxillin and activation of Rac1 $1^{14,15,17}$. Our results demonstrated that ectopic expression of miR-141 suppressed CDKN2B-AS1 and cyclin-D1/D2 expression in RCC cell lines. We also observed a decrease in phospho-paxillin and Rac1 in miR-141 overexpressing cells compared to controls. Elevated paxillin phosphorylation is common in cancer tissues and is associated with tumorigenesis, EMT, and metastasis ${ }^{15,16,50}$. Paxillin interacts with numerous molecules, controlling the Rho family of GTPases, that are crucial regulators of adhesion dynamics ${ }^{16,51}$. Rac1 GTPase is required for cell migration and its hyperactivation results in cancer invasiveness and progression ${ }^{52}$. Similarly, we also found that knockdown of $C D K N 2 B-A S 1$ reduced cyclin-D1/D2 expression, thereby decreasing phosphorylation of paxillin and activation of rac1 to inhibit migration/invasion. Thus, CDKN2B-AS1 functions as a ceRNA for miR-141 promoting cyclin-D1/D2 expression, resulting in RCC aggressiveness.

In summary, our study documents novel interactions between $C D K N 2 B-A S 1 / \mathrm{miR}-141$ and cyclin D1-D2 in RCC. In view of our present in vitro and in vivo data, $C D K N 2 B-A S 1$, cyclin-D1, and cyclin-D2 are shown to be direct and functional targets of miR-141 contributing to EMT by regulating rac1 and phospho-paxillin expression. Collectively, CDKN2B-AS1/miR-141 is identified as a novel interaction regulating tumorigenic progression and metastasis in RCC through the cyclin-D/Rac/paxillin network, and thus can be an attractive target for therapeutic intervention of aggressive RCC. 


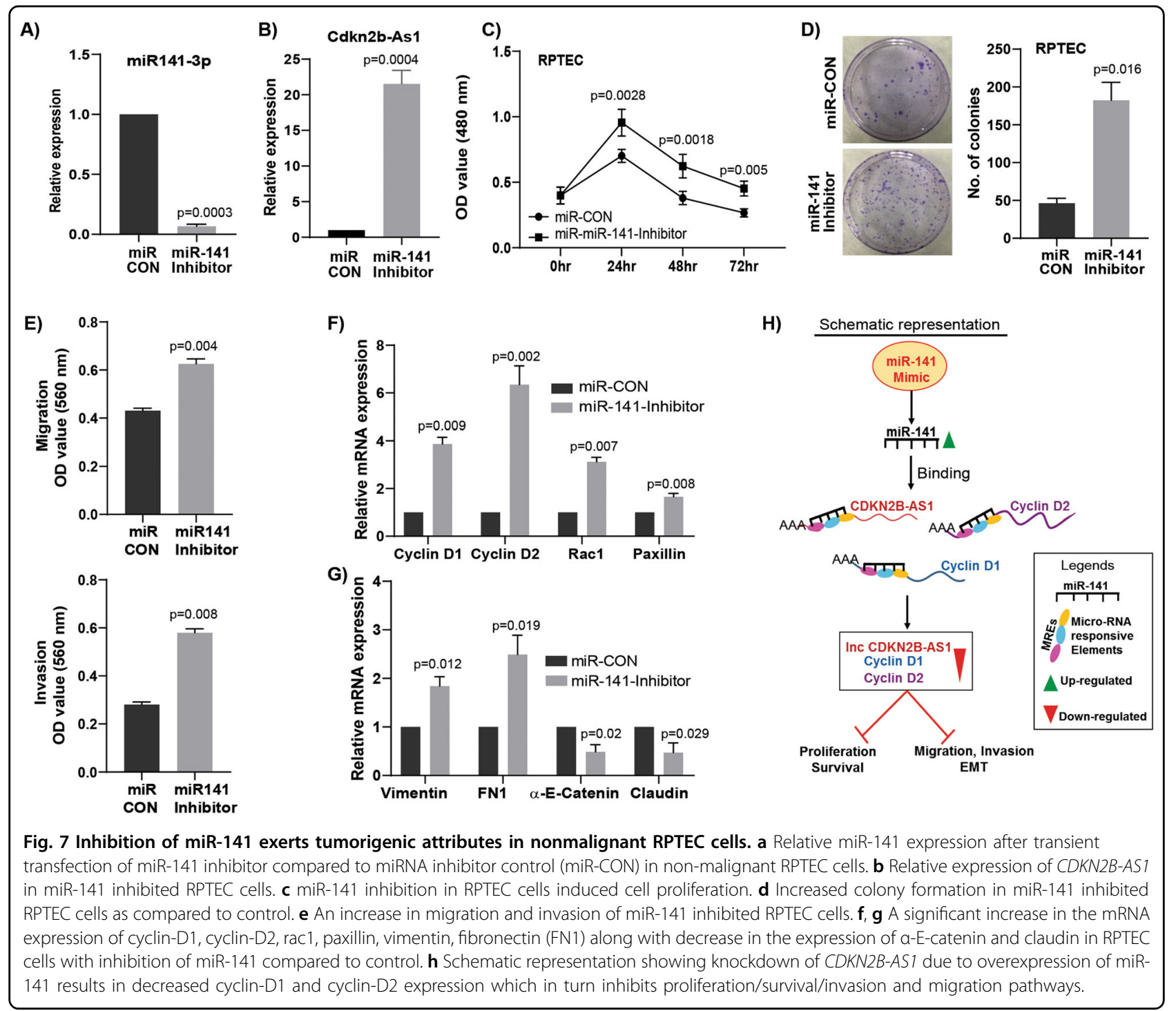

\section{Acknowledgements}

We thank Dr. Roger Erickson for his support and assistance with the preparation of the paper.

\section{Author details}

'Department of Urology, Veterans Affairs Medical Center, San Francisco and University of California San Francisco, San Francisco, CA, USA. ${ }^{2}$ Department of Surgery, University of Miami Miller School of Medicine, Miami, FL, USA.

${ }^{3}$ Medical College of Georgia, Augusta University, Augusta, GA, USA

\section{Conflict of interest}

The authors declare that they have no conflict of interest.

\section{Publisher's note}

Springer Nature remains neutral with regard to jurisdictional claims in published maps and institutional affiliations.

Supplementary Information accompanies this paper at (https://doi.org/ 10.1038/s41419-020-02877-0).
Received: 9 April 2020 Revised: 2 July 2020 Accepted: 6 July 2020

Published online: 19 August 2020

\section{References}

1. Siegel, R. L., Miller, K. D. \& Jemal, A. Cancer statistics, 2020. Ca. Cancer J. Clin. 70, 7-30 (2020).

2. Zlotta, A. R. The changing natural history of renal cell carcinoma. J. Urol. 166, 1611-1623 (2001)

3. McDermott, D. F. et al. Randomized phase III trial of high-dose interleukin-2 versus subcutaneous interleukin-2 and interferon in patients with metastatic renal cell carcinoma. J. Clin. Oncol. 23, 133-41 (2005).

4. Belldegrun, A. S. et al. Cancer-specific survival outcomes among patients treated during the cytokine era of kidney cancer (1989-2005): a benchmark for emerging targeted cancer therapies. Cancer 113, 2457-63 (2008).

5. Rydzanicz, M., Wrzesiński, T., Bluyssen, H. A. R. \& Wesoły, J. Genomics and epigenomics of clear cell renal cell carcinoma: Recent developments and potential applications. Cancer Lett. 341, 111-126 (2013).

6. Majid, S. et al. MicroRNA-205 inhibits Src-mediated oncogenic pathways in renal cancer. Cancer Res. https://doi.org/10.1158/0008-5472.CAN-10-3666 (2011). 
7. Brown, R. L. et al. CD44 splice isoform switching in human and mouse epithelium is essential for epithelial-mesenchymal transition and breast cancer progression. J. Clin. Invest. 121, 1064-1074 (2011).

8. De, S., Das, S., Mukherjee, S., Das, S. \& Sengupta (Bandyopadhyay), S. Establishment of twist-1 and TGFBR2 as direct targets of microRNA-20a in mesenchymal to epithelial transition of breast cancer cell-line MDA-MB-231. Exp. Cell Res. 361, 85-92 (2017).

9. Lamouille, S., Xu, J. \& Derynck, R. Molecular mechanisms of epithelial-mesenchymal transition. Nat. Publ. Gr. https://doi.org/10.1038/ nrm3758 (2014).

10. Prieto-García, E., Díaz-García, C. V., García-Ruiz, I. \& Agulló-Ortuño, M. T. Epithelial-to-mesenchymal transition in tumor progression. Med Oncol 34, 122 (2017).

11. De Craene, B. \& Berx, G. Regulatory networks defining EMT during cancer initiation and progression. Nat. Rev. Cancer 13, 97-110 (2013).

12. Bhat, N. S. et al. MicroRNA-720 regulates E-cadherin-aE-catenin complex and promotes renal cell carcinoma. Mol. Cancer Ther. 16, 2840-2848 (2017).

13. Watabe, M., Nagafuchi, A., Tsukita, S. \& Takeichi, M. Induction of polarized cellcell association and retardation of growth by activation of the E-cadherincatenin adhesion system in a dispersed carcinoma line. J. Cell Biol. 127, 247-256 (1994)

14. Fusté, N. P. et al. Cytoplasmic cyclin D1 regulates cell invasion and metastasis through the phosphorylation of paxillin. Nat. Commun. 7, 11581 (2016).

15. Fust, N. P., Ferrezuelo, F. \& Gar, E. Cyclin D1 promotes tumor cell invasion and metastasis by cytoplasmic mechanisms. Mol. Cell. Oncol. 3, e1203471 (2016).

16. Montalto, F. I. et al. Progesterone receptor B signaling reduces breast cancer cell aggressiveness: role of Cyclin-D1/Cdk4 mediating paxillin phosphorylation. Cancers (Basel). https://doi.org/10.3390/cancers11081201 (2019).

17. Li, Z. et al. Cyclin D1 induction of cellular migration requires p27 KIP1. Cancer Res. https://doi.org/10.1158/0008-5472.CAN-06-1596 (2006).

18. Dasgupta, P. et al. MicroRNA-203 inhibits long noncoding RNA hotair and regulates tumorigenesis through epithelial-to-mesenchymal transition pathway in renal cell carcinoma. Mol. Cancer Ther. 17, 1061-1069 (2018).

19. Ruvkun, G. MOLECULAR BIOLOGY: Glimpses of a Tiny RNA World. Science 294 797-799 (2001).

20. Bartel, D. P. MicroRNAs: Genomics, Biogenesis, Mechanism, and Function. Cell 116, 281-297 (2004)

21. Wang, K. C. \& Chang, H. Y. Molecular mechanisms of long noncoding RNAs. Mol Cell. https://doi.org/10.1016/j.molcel.2011.08.018 (2011).

22. Esteller, M. Non-coding RNAs in human disease. Nat. Rev. Genet. 12, 861-874 (2011).

23. Huarte, M. \& Rinn, J. L. Large non-coding RNAs: missing links in cancer? Hum. Mol. Genet. https://doi.org/10.1093/hmg/ddq353 (2010).

24. Cesana, M. et al. A long noncoding RNA controls muscle differentiation by functioning as a competing endogenous RNA. Cell 147, 358-369 (2011).

25. Ergun, S. \& Oztuzcu, S. Oncocers: ceRNA-mediated cross-talk by sponging miRNAs in oncogenic pathways. Tumor Biol. 36, 3129-3136 (2015).

26. Wang, Q. et al. Long non-coding RNA HOTTIP promotes renal cell carcinoma progression through the regulation of the miR-615/IGF-2 pathway. Int. J. Oncol. 53, 2278-2288 (2018).

27. Díez-Villanueva, A., Mallona, I. \& Peinado, M. A. Wanderer, an interactive viewer to explore DNA methylation and gene expression data in human cancer. Epigenetics Chromatin 8, 22 (2015).

28. Nip, $\mathrm{H}$. et al. Oncogenic microRNA-4534 regulates PTEN pathway in prostate cancer. Oncotarget 7, 68371-68384 (2016).

29. Fu, R. J. et al. DNMT1-maintained hypermethylation of Krüppel-like factor 5 involves in the progression of clear cell renal cell carcinoma. Cell Death Dis. 8 e2952 (2017)
30. Trang, $P$. et al. Regression of murine lung tumors by the let-7 microRNA Oncogene 29, 1580-1587 (2010).

31. Kulkarni, P. et al. Elevated miR-182-5p associates with renal cancer cell mitotic arrest through diminished MALAT-1 expression. Mol. Cancer Res. 16, 1750-1760 (2018).

32. Arvey, A., Larsson, E., Sander, C., Leslie, C. S. \& Marks, D. S. Target mRNA abundance dilutes microRNA and siRNA activity. Mol. Syst. Biol. 6, 363 (2010).

33. Ebert, M. S., Neilson, J. R. \& Sharp, P. A. MicroRNA sponges: competitive inhibitors of small RNAs in mammalian cells. Nat. Methods 4, 721-726 (2007).

34. Poliseno, L. et al. A coding-independent function of gene and pseudogene mRNAs regulates tumour biology. Nature 465, 1033-1038 (2010).

35. Sumazin, P. et al. An Extensive MicroRNA-Mediated Network of RNA-RNA Interactions Regulates Established Oncogenic Pathways in Glioblastoma. Cell 147, 370-381 (2011).

36. Chen, S. et al. The over expression of long non-coding RNA ANRIL promotes epithelial-mesenchymal transition by activating the ATM-E2F1 signaling pathway in pancreatic cancer: An in vivo and in vitro study. Int. J. Biol. Macromol. 102, 718-728 (2017).

37. Qiu, J. J. et al. The long non-coding RNA ANRIL promotes proliferation and cell cycle progression and inhibits apoptosis and senescence in epithelial ovarian cancer. Oncotarget 7, 32478-32492 (2016).

38. Cui, X., Yu, T., Shang, J., Xiao, D. \& Wang, X. Long non-coding RNA CDKN2BAS1 facilitates laryngeal squamous cell cancer through regulating miR-497/ CDK6 pathway. Onco. Targets Ther. 12, 8853-8862 (2019).

39. Yang, X. \& Wang, P. MiR-188-5p and MiR-141-3p influence prognosis of bladder cancer and promote bladder cancer synergistically. Pathol. Res. Pract. 215, 152598 (2019)

40. Richardsen, E. et al. MicroRNA 141 is associated to outcome and aggressive tumor characteristics in prostate cancer. Sci. Rep. 9, 386 (2019).

41. Ding, L., Yu, L. L., Han, N. \& Zhang, B.T. miR-141 promotes colon cancer cell proliferation by inhibiting MAP2K4. Oncol. Lett. 13, 1665-1671 (2017).

42. Du, Y. et al. Down-regulation of miR-141 in gastric cancer and its involvement in cell growth. J. Gastroenterol. 44, 556-561 (2009).

43. Braun, J., Hoang-Vu, C., Dralle, H. \& Hüttelmaier, S. Downregulation of microRNAs directs the EMT and invasive potential of anaplastic thyroid carcinomas. Oncogene 29, 4237-4244 (2010).

44. Chiyomaru, T. et al. Long non-coding RNA hotair is targeted and regulated by MIR-141 in human cancer cells. J. Biol. Chem. 289, 12550-12565 (2014).

45. Bert, S. A. et al. Regional activation of the cancer genome by long-range epigenetic remodeling. Cancer Cell 23, 9-22 (2013).

46. Heinzelmann, J. et al. Specific miRNA signatures are associated with metastasis and poor prognosis in clear cell renal cell carcinoma. World J. Urol. 29, 367-73 (2011).

47. Allegra, A. et al. Circulating microRNAs: New biomarkers in diagnosis, prognosis and treatment of cancer. Int. J. Oncol. 41, 1897-1912 (2012).

48. Ye, J. U. N. et al. MicroRNA-141 inhibits tumor growth and minimizes therapy resistance in colorectal cancer. Mol Med Rep. 15, 1037-1042 (2017).

49. Wang, Y. microRNA-1297 inhibits the growth and metastasis. DNA Cell Biol. $\mathbf{3 6}$ 991-999 (2017).

50. Brown, M. C. \& Turner, C. E. Paxillin: Adapting to change. Physiological Rev. 84, 1315-1339 (2004)

51. Tang, K., Boudreau, C. G., Brown, C. M. \& Khadra, A. Paxillin phosphorylation at serine 273 and its effects on Rac, Rho and adhesion dynamics. PLoS Comput. Biol. 14, e1006303 (2018)

52. Seiz, J. R. et al. Different signaling and functionality of Rac1 and Rac1b in the progression of lung adenocarcinoma. Biol. Chem. 401, 517-531 (2019). 